Research Article

\title{
Index cohesive force analysis of the U.S. stock market
}

\author{
D. Y. Kenett ${ }^{1}$, Y. Shapira ${ }^{1}$, G. Gur-Gershgoren ${ }^{2,3}$, and E. Ben-Jacob ${ }^{1, *}$ \\ ${ }^{I}$ School of Physics and Astronomy, Tel-Aviv University, Tel-Aviv, Israel. \\ ${ }^{2}$ School of Business and Management, Ben Gurion University, Beer Sheva 84105, Israel. \\ ${ }^{3}$ Dep. of Economic Research, Israel Securities Authority, 22 Kanfei Nesharim St., Jerusalem 95464, Israel.
}

\begin{abstract}
The 2007-2009 financial crisis, and its fallout, has strongly emphasized the need to define new ways and measures to study and assess the dynamics of financial markets. The S\&P500 dynamics during 4/1999-4/2010 is investigated in terms of the index cohesive force (ICF - the balance between the stock correlations and the partial correlations after subtraction of the index contribution). We found a rapid market transition at the end of 2001 from a flexible state of low ICF into a stiff (nonflexible) state of high ICF that is prone to market systemic collapses. The stiff state is also marked by strong effect of the market index on the stock-stock correlations as well as bursts of high stock correlations reminiscence of epileptic brain activity. Finally, we make use of the recently introduced stock dependency networks to uncover changes in the market structure following the transition at the end of 2001. This analysis sheds new light on the origin and nature of the current crisis. The new approach is likely to be applicable to other classes of complex systems from gene networks to the human brain.
\end{abstract}

Keywords: ICF analysis, stock correlations, Residual correlation.

\section{Introduction}

The current financial crisis began with the collapse of the subprime bubble at the end of 2007 [1,2], and then spread to the global financial markets and economies worldwide. In the past, and in the aftermath of the crisis, much work has been devoted to the study and characterization of financial bubbles [1, 3-9].

In a recent study, Sornette et al. [1] have presented a general framework in which they propose that the fundamental cause of the crisis was in fact an accumulation of several bubbles in the markets, and the interplay between these bubbles. The formation of bubbles in the markets is followed by a strong herding phenomenon amongst traders [9], and the burst of these bubbles is accompanied by strong synchrony in the markets reminiscent of epileptic seizures. For example, Lillo et al. [10, 11] have investigated the dynamics of markets following crashes. Such synchrony in the markets can be used as a predictive measure for the formation of bubbles, and more importantly, for the burst of such bubbles. As such, it is crucial to develop new quantitative measures to fully capture, characterize and understand the market dynamical states, stability and transition between economic states. Currently, in this regard, much work is focused on the analysis of zero lagged [12] or higher-order lagged correlations [13], a detrneding approach to the study of cross correlations [14-16], and other measures to study co-movement and synchronization in stock markets $[17,18]\}$

* E-mail address: eshelbj@gmail.com

ISSN: 1791-2377 @ 2011 Kavala Institute of Technology. All rights reserved.
Recently, we have presented a new, physics motivated, analysis framework to investigate the dynamics of markets, during the past decade[19]. We show that the fragility of the market could be detected as early as the beginning of 2002, when the market dynamics went through a rapid change that was marked by a jump in the index cohesive force (ICF), and a decline in the correlation Eigenvalue entropy. This transition in the market dynamical state created a significant change in the structure of the market, due to an abnormal dominance of the market index on the stock correlations. The outcome was a rapid transition into a stiff market state that lacked a sufficient degree of freedom and internal flexibility of response to extreme changes. Hence, the index dominance rendered the market prone so systemic collapses as in the case of the sub-prime crisis.

We investigated the time dynamics of the S\&P500 index, and 418 of its constituting stocks (not all 500 stocks were traded for the entire time period), during the last decade from April 1999 to April 2010 (see also Text S1, for full description of the dataset). The investigations were carried out in terms of the index cohesive force (ICF) - the balance between the raw stock correlations that include the index effect and the residual stock correlations (or partial correlations) after subtraction of the index effect [20,21]. The ICF provides a means to identify structural changes in the market, which significantly alter the stability of these markets.

Here we expand the work presented by Kenett et al. [19], by studying the change in the structure of the market following the transition observed at the end of 2001. To this end, we make use of the stock dependency network 
methodology [22] to investigate the stock dependency relationships before and following the transition.

\section{Methods}

\subsection{Raw Stock Correlations}

The similarity between stock price changes is commonly calculated by the Pearson's correlation coefficient [21]. The raw stock correlations [21, 23] are calculated for time series of the log of the daily return, given by:

$$
r_{i}(t)=\log \left[P_{i}(t)\right]-\log \left[P_{i}(t-1)\right]
$$

Where $P_{i}(t)$ is the daily adjusted closing price of stock $i$ at day $t$. The raw stock correlations are calculated using the Pearson's correlation coefficient $C(i, j)$ between every pair of stocks $i$ and $j$, where

$C(i, j)=\frac{\left\langle\left(r_{i}-\left\langle r_{i}\right\rangle\right) \cdot\left(r_{j}-\left\langle r_{j}\right\rangle\right)\right\rangle}{\sigma_{i} \cdot \sigma_{j}}$

\langle\rangle denotes average, and $\sigma$ are the standard deviations (STD).

\subsection{Residual correlation}

Recently, we have made use of partial correlations to calculate the residual correlation between stocks, after removing the affect of the index [21]. Partial correlation is a powerful tool to investigate how the correlation between two stocks depends on the correlation of each of the stocks with a third mediating stock or with the index as is considered here. The residual, or partial, correlation $\rho(i, j \mid m)$ between stocks $i$ and $j$, using the Index $(m)$ as the mediating variable is defined by $[20,21,24]$

$$
\rho(i, j \mid m)=\frac{C(i, j)-C(i, m) \cdot C(j, m)}{\sqrt{\left(1-C^{2}(i, m)\right) \cdot\left(1-C^{2}(j, m)\right)}}
$$

Note that according to this definition, $\rho(i, j \mid m)$, can be viewed as the residual correlation between stocks $i$ and $j$, after subtraction of the contribution of the correlation between each of the stocks with the Index.

\subsection{Index Cohesive Force}

In the past, we have shown that the market index has a cohesive effect on the dynamics of the stock correlations [21]. This refers to the observed affect the index has on stock correlations, where we have found that larger changes of the index result in higher stock correlations, and as such more cohesive [21]. Here we expand this analysis and introduce a quantitative measure of the index cohesive force. We define $\operatorname{ICF}(\tau)$ - the index cohesive force calculated over a time window $\tau$, as a measure of the balance between the raw and residual correlations given by,

$$
\operatorname{ICF}(\tau)=\frac{\overline{\langle C(i, j)\rangle_{\tau}}}{\overline{\langle\rho(i, j \mid m)\rangle_{\tau}}}
$$

where $\tau$ the time window, during which the average correlation and average residual correlation are calculated, denoted by $\overline{\langle C(i, j)\rangle_{\tau}}\left(\overline{\langle P C(i, j \mid m)\rangle_{\tau}}\right)$ is the average of average correlation (partial correlation).

\subsection{Stock dependency networks}

The dependency network approach provides a new system level analysis of the activity and topology of directed networks. The approach extracts causal topological relations between the network's nodes, and provides an important step towards inference of causal activity relations between the network nodes.

The relative effect of the correlations $C(i, j)$ and $C(j, k)$ of node $j$ on the correlation [22] is given by:

$d(i, k \mid j) \equiv C(i, k)-P C(i, k \mid j)$

We note that this quantity can be viewed either as the correlation dependency of $C(i, k)$ on node $j$ (the term used here), or as the correlation influence of node $j$ on the correlation $C(i, k)$.

Next, we define the total influence of node $j$ on node $i$, or the dependency $D(i, j)$ of node $i$ on node $j$ to be:

$$
D(i, j)=\frac{1}{N-1} \sum_{k \neq j}^{N-1} d(i, k \mid j)
$$

As defined, $D(i, j)$ is a measure of the average influence of node $j$ on the correlations $C(i, k)$, over all nodes $k$ not equal to $j$. The node activity dependencies define a dependency matrix $D$ whose $(i, j)$ element is the dependency of node $i$ on node $j$. It is important to note that while the correlation matrix $C$ is a symmetric matrix, the dependency matrix $D$ is nonsymmetrical $-D(i, j) \neq D(j, i)$ since the influence of node $j$ on node $i$ is not equal to the influence of node $i$ on node $j$. Once the dependency matrix is constructed, we use the Planar Maximally Filtered Graph (PMFG) methodology [22] to construct the stock dependency network.

\section{Results}

The average raw correlation between stocks has been investigated in the past [25-28], with the focus being on large time windows (200 to 500 days) to reduce the statistical variations. Here we selected a shorter, 22 trading days (corresponding to one work month), time window. We validated that while these short time windows retained limited variations (as shown by the results), they are successful in capturing short time events in the market dynamics. Such short time localized events are averaged out and cannot be deciphered when long time windows are used. In particular, we will show that using these short time 
windows enabled us to reveal changes in the index cohesive force that are very rapid and of high magnitude.

\subsection{Time dynamics of the raw and residual correlations and market stiffness}

We begin our investigation by studying the dynamics of the stocks' raw correlations (Figure 1B) and residual correlations (Figure 1C), in comparison to the dynamics of the S\&P500 index (Figure 1A). Such analysis reveals a transition in the market, taking place at the end of 2001. Following the transition, the market entered into a state dominated by the index as is reflected by the very small residual correlations in the new dynamical state. This state is characterized by an abnormal dominance of the market index, and a state in which the effect other processes such as the influence of different economic sectors is drastically reduced. We propose, in light of the recent global financial events, that the outcome is that the strong index influence rendered the market into a stiff state that is less adaptable to financial changes and therefore is more prone to crises. In other words, being a complex system [21, 29], when the average interactions between the market stocks becomes very large, the market becomes inflexible and more sensitive to external changes and thus more prone to crises.
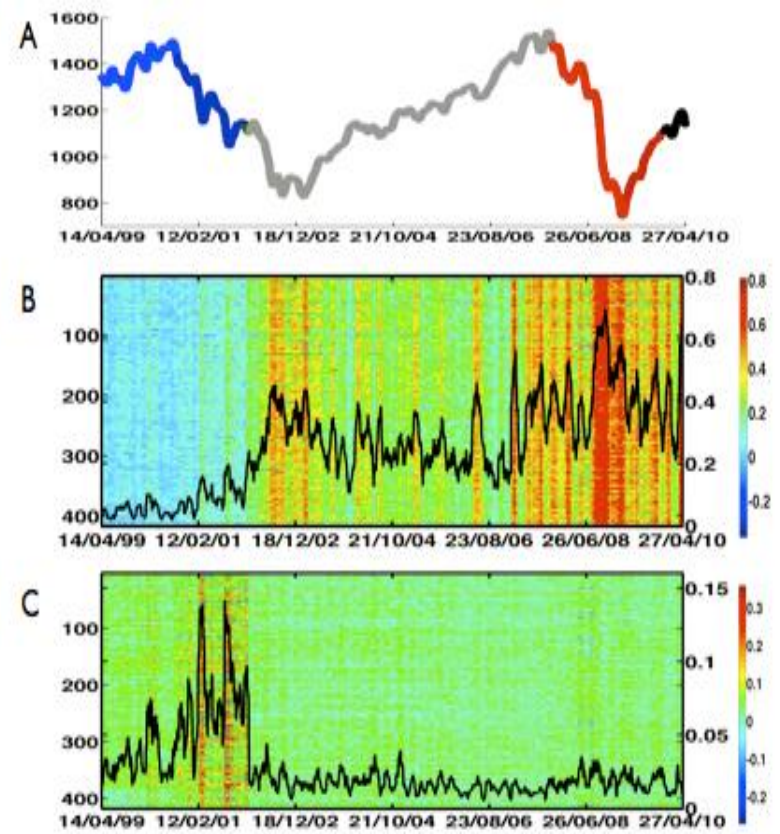

Fig. 1. The dynamics of correlations and partial correlations for stocks belonging to the S\&P500 Index from April 1999 till April 2010, versus the price of the Index. A) The S\&P500 market index from April 1999 until the end of April 2010 Different time periods are marked by different colors: blue - April 1999 to December 2001, green December 2001 to January 2002 (transition period), grey - January 2002 to July 2007, light red - August 2007 - March 2009 (crisis), red March 2009 - January 2010 (recovery), and black - January 2010 to April 2010; B) Raster plot of the stock raw correlations, calculated according to the stocks daily returns and for 22 trading days windows. Each row shows the averaged correlations of a specific stock with all other stocks (left y axis), with the mean stock raw correlations (over all the stock correlations) superimposed in black (right y axis). C) Raster plot of the stock residual correlations after subtracting the index contribution, with the mean market residual correlations superimposed in black. In panel A the different colors indicate different time periods. In panels $B$ and $C$ the colors of the raster plots represent the strength of the correlations, as indicated in the color bars at the right side of each plot.
The anomalous dominance of the index and the market dangerous stiffness of this market state since the end of 2001 , is manifested by the emergence of market seizure-like behavior - bursts of very high stock raw correlations that usually coincide with local minima in the index (Figure 1B). Performing our analysis using longer time windows resulted in qualitatively similar results, in which the transition in the market was still captured, while the localized bursts of correlation were no longer present.

\subsection{Dynamics of the index cohesive force}

In Figure 2 we plot the ICF as a function of time. Studying the dynamics of the ICF, it is easy to observe the transition at the end of 2001 (see Figure 2), which is also visible in panels $\mathrm{B}$ and $\mathrm{C}$ of Figure 1. This transition occurs during the month of December 2001. Following this transition, we observe strong fluctuations in the iCF. It is noteworthy that for the period of April-September 2008 the ICF was at low values, with small fluctuations, which is followed by a strong jump following the Lehman Brothers bankruptcy.

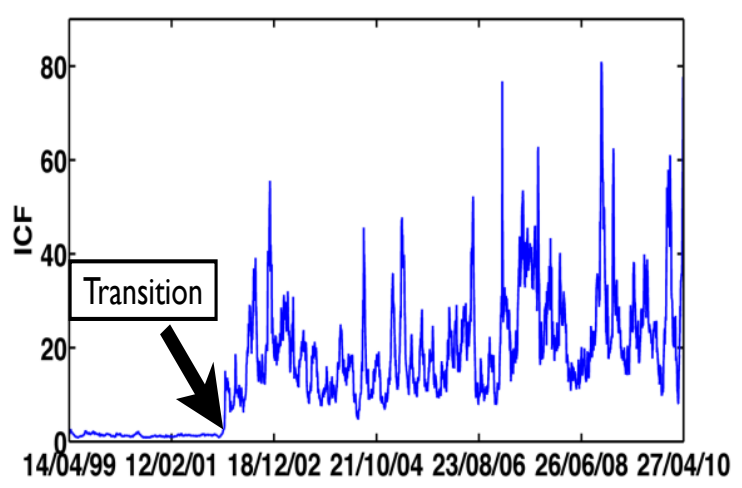

Fig. 2. The ICF for the S\&P500 stocks, as a function of time, for the period of $1999-2010$

In Figure 3 we present the time evolvement of the ICF, versus the average stocks-index correlations. In the left panel we use the same coloring scheme as in Figure 1A. The results well depict the significant difference between the two market states. In the right panel of Figure 3, we highlight the time period of 2010, using a color scheme from light yellow at the beginning of the year to black at the end of April. Using this color code, we observe that during early 2010 the market dynamics moved back towards the stable state, but this trend was reversed at the end of March.

To further assess the current state of the market, we calculated the ICF for the entire year of 2010. In Figure 4A we present the time evolvement of the ICF for 2010. We divide the entire year into 5 periods, based on the changes in the ICF. As was observed in Figure 3B, we find a drop in the ICF at the beginning of 2010 (blue circle), followed by a dramatic jump in the ICF (green circle). In addition to the strong peak in the ICF observed for April 2010, we observe additional somewhat weaker peaks, in June and August of 2010. Finally, as presented in Figure 3B, we compare the ICF to the average stock-index correlation, for the entire year of 2010 (Figure 4B, color coded according to Figure $4 \mathrm{~A})$. We note that in general, the year of 2010 was dominated by high values of the ICF, which remains high at the end of the year. Furthermore, comparing Figure 4B to 
Figure $3 \mathrm{~A}$, we observe that the market is still in the abnormal stiff state so it continues to be prone to systemic collapses.
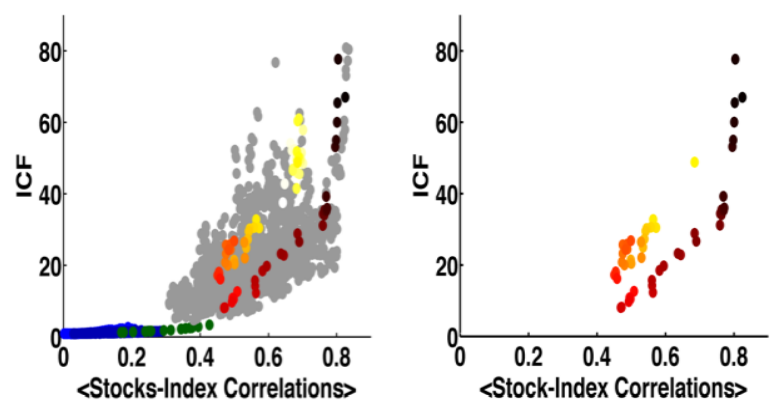

Fig. 3. Time evolvement of the S\&P500 market index cohesive force (ICF - the ratio between the raw correlations and the bare (partial) correlations), as function of the stocks-index correlations, during the last decade. The color code on the left is as in Figure 1A in the text. On the right, we only present the time progression during 2010, colored from light yellow at the beginning of the year to black at the end of April. Using this color code, we observe that during early 2010 the market dynamics moved back towards the stable state, but this trend was reversed at the end of March and currently the market instability seems to rapidly evolve towards a more fragile state.
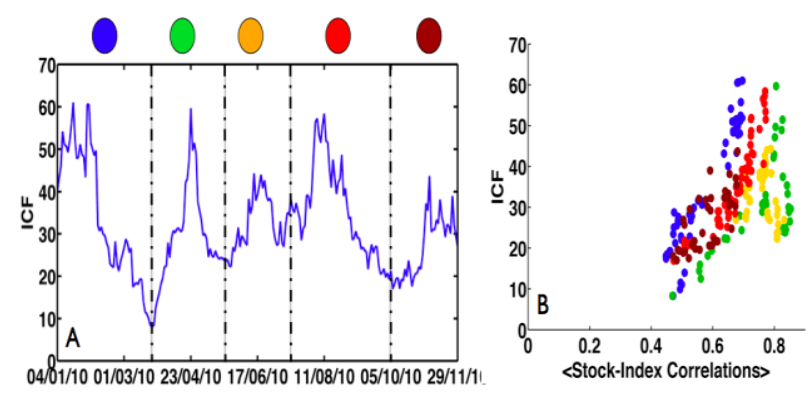

Fig.4. Time evolvement of the ICF for the entire year of 2010. (A) The ICF as a function of time, for 2010. We observe that the fluctuations of the ICF during 2010 were strong; we identify 5 different periods, which are characterized by changes in trend of the ICF. The Transition from the first period (blue circle) to the second (green circle) is similar to the one presented in Figure 2B. Furthermore, we observe two more strong peaks in the ICF - at June and in August of 2010. (B) Comparison of the ICF to the average stock index correlation, as presented in Figure 3, for the entire year of 2010. Color code as is indicated in Figure 4A.

\subsection{Manifestation of the transition at the end of 2001}

The dramatic differences between the flexible and stiff (inflexible) market states are best manifested in the 3dimensional scatter plot presented in Figure 5A. The axes of this $3 \mathrm{D}$ space are the average Stocks-Index correlations, the average raw correlations, and the average residual correlations. The color code makes transparent the fact that the market dynamical state was not determined by the Index trend (positive or negative): The stiff state started in the midst of a decline in the Index and continued unchanged as the Index trend changed several times. To demonstrate this change, we show in Figure 5B a scatter plot in a different 3D space - the axes are the spectral entropy SE, the average beta coefficient, $\left\langle\beta_{i}\right\rangle$ and the average residual correlations[19]. Clearly the two scatter plots capture the same phenomenon.
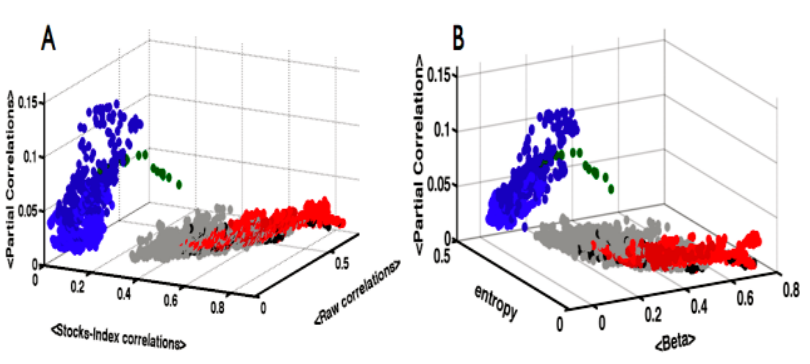

Fig. 5. A three-dimensional scatter plot of the market dynamical evolution of stocks belonging to the S\&P500 index in the past decade. (A) The axes are the average stocks-index correlations (X-axis), average raw stock correlations (Y-axis), and the average residual (partial) correlations (Z-axis). Each dot corresponds to a time window of 22 trading days and the color code is similar to that used in Figure 1A. (B) similar results are obtained when using longer time window and when replacing the average stocks-index correlation with the average

coefficient as the $\mathrm{X}$-axis, and replacing the average stock-stock correlation with the entropy as the $\mathrm{Y}$-axis.

In Figure 6, we re-plot the price of the S\&P500 index, the average stock correlation, and the average partial correlation, as a function of time. We emphasize the transition, and the two different states observed for the market. Here we focus on the period of March 2000 to March 2011.

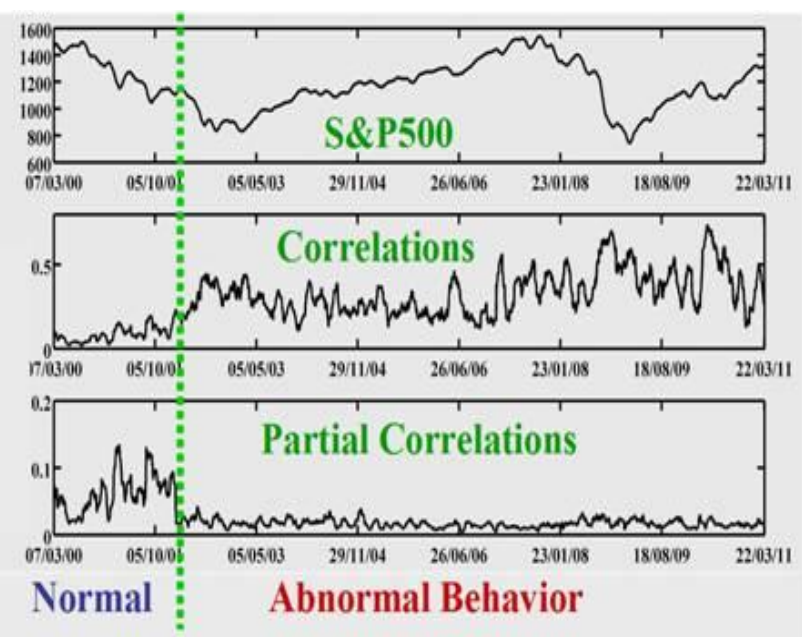

Figure 6: Price of the S\&P500 index (top), average stock correlation (middle) and average partial correlation (bottom) as a function of time, for the period of March 2000 - March 2011. We emphasize the transition and the two different market states.

\subsection{Changes in the structure of the market}

Analyzing the dynamics of correlations in the U.S. market using the ICF analysis revealed two distinct market states, as discussed above. To investigate these two market states, we make use of the stock dependency network methodology [22].

To investigate the structure of the market at each state, we focus on three sectors - the Financial sector $(n=64$ stocks), Industrial sector ( $\mathrm{n}=58$ stocks), and Information Technology sector (IT, $\mathrm{n}=63$ stocks). We then construct the dependency network for state I (corresponding to the period 1999-2001) and for state II (corresponding to the period 2002-2011) separately. 
In Figure 7 we present the dependency networks for state I (left) and state II (right). Each sector is marked by a given color: Financial sector - red, Industrial sector - green, and IT sector - blue. For state I, the structure of the network is more modular, and each sector mainly influences itself. In stark contrast, in state II the network structure breaks down and the network loses its modularity. This result emphasizes the change in the market following the transition observed at the end of 2001 .
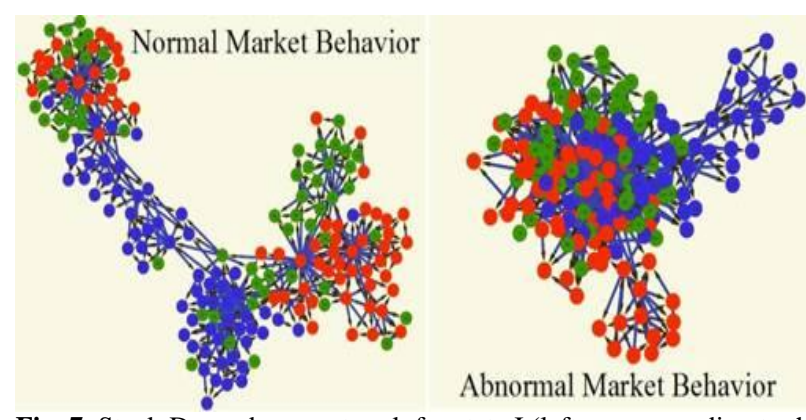

Fig. 7. Stock Dependency network for state I (left, corresponding to the period 1999-2001), and state II (right, corresponding to the period 20022011). The network is constructed for three sectors: Financial sector (n $=64$ stocks, red $)$, Industrial sector $(n=58$ stocks, green $)$ and Information Technology sector $(\mathrm{n}=63$ stocks, blue).

\section{Discussion}

In summary, we presented new approaches to quantify the dynamics of the stock market, using the correlation entropy and the index cohesive force (ICF). The ICF parameter provides a new quantitative measure to investigate different financial states of the market, and the transitions between these states. Once the different market states are identified, different tools can be applied to study the difference in market structure in each state. Here we make use of the dependency network methodology, and uncover unchanges in market structure.

Using this approach we discovered a rapid transition in the market dynamical state at the end of 2001. This transition is manifested by a jump in the stock correlations, and a sharp fall in the stock residual correlations. After the transition the market entered into a high ICF stiff state. In this state the index predominantly affects the market dynamics while it shades the effect of other degrees of freedom that can contribute to the market flexibility.

Thus, we suggest that during this state the market is highly prone to systematic collapses, even due to relatively small external perturbations, leaving it incapable of coping with crises. This interpretation is consistent with the fact that following the burst of the subprime bubble and the fall of Lehman Brothers [30, 31], the market collapsed. It is also reasonable to assume that this rapid transition at the end of 2001 might have been a consequence of the "dot-com" bubble crisis, combined with the traumatic events which took place in the U.S. at the beginning of the decade and the outcome of the rapid interest cuts [32] and other financial policies employed to overcome the fallout effect of those. One such important financial policy was the implementation of the Decimal Pricing system in the American stock markets. The process of implementation was finalized in the NYSE at January 2001, and in the NASDAQ at April 2001. However, the observed transition in the market uncovered by the ICF took place at December 2001; thus, this change in tick size is one more contributing factor to the transition in the market.

The time period studied here covers the two largest crises that took place in the past decade - the 2000-2001 ".com" crisis, and the 2007-2009 credit crunch crises. During the ".com" period, internet and technological companies were hit hard by the crisis, while other sectors were less affected. This was a local crisis, and the bubblecrash was unevenly distributed among these sectors. This means that the residual correlations during this period should be unusually high, as indeed we found. The credit crunch crisis was a systemic (global) one, which spilled over from the financial sector into all other sectors. As such, the entire market dynamics exhibited high synchrony, as is reflected by the high values of the ICF measure introduced here. As we have shown, during the first part of 2010 there seemed to be a recovery in the markets, which was accompanied by a drop in the values of the ICF. However, a jump in the ICF, and indeed a renewed dangerous process in the market followed this drop in late March. Extending the analysis of the ICF to the entire year of 2010, we find that the ICF remains high; furthermore, short periods of relaxation in the ICF are followed by strong jumps in the ICF. Finally, we find that the end of 2010 is marked by an upwards trend in the ICF, which shows that the market is still in the abnormal state, and still strongly prone to systematic collapse.

Finally, we investigate the structure of the market for the two observed states. To this end, we make use of the stock Dependency Network methodology [22]. We focus on the three main sectors - the Financial, Industrial, and Information Technology (IT) sector - and study the dependency network constructed for each state. This methodology provides important hidden information on the dependence relationships in the system, such as the dependence of a given stock on other stocks, or the dependence of a given sector on other sectors. This analysis clearly reveals the change in the market structure, following the transition at the end of 2001. Prior to the transition (state I), the network is very modular, where the influence is mainly contained in each individual sector. Following the transition (state II), the modularity of the network breaks down, and the network becomes highly mixed, with strong cross-sectorial influence. Furthermore, we observe that in the second state, the Financial sector is central in the network, which is in agreement with our previous findings [22] and also with the findings presented above (see also [19]), which indicate that the U.S. market has had a transition into a market dominated by the Financial sector.

In conclusion, we propose the ICF as a new system-level parameter, which provides an efficient measure used to describe and quantify the market dynamical state, and which can be used as a tool to monitor the stability of stock markets. The stability of the markets is crucial for the world's economies, thus this tool can be very important to governments and regulation agencies worldwide. Future work includes expanding this analysis to other markets, and to make use of this parameter as an early-warning mechanism for changes in the state of the market.

\section{Acknowledgments}

We thank Rosario Mantegna, and Didier Sornette for their insights and helpful conversations on the subject. We also thank Sorin Solomon for his illuminating comments on the results presented in this study. This research has been 
supported in part by the Tauber Family Foundation and the Maguy-Glass Chair in Physics of Complex System at the Tel
Aviv University

\section{References}

1. D. Sornette and R. Woodard, Financial Bubbles, Real Estate Bubbles, Derivative Bubbles, and the Financial and Economic Crisis, Econophysics Approaches to Large-Scale Business Data and Financial Crisis: 10.1007/978-4-431-53853-0_6, 101 (2010).

2. J.H. Boyd, R. Jagannathan and S. Kwak, What Caused the Current Financial Mess and What Can We Do about It? (Digest Summary), CFA Digest, 40 (2) (2010).

3. K. Bastiaensen, P. Cauwels, D. Sornette, R. Woodard and W. Zhou, The Chinese equity bubble: Ready to burst, Quantitative Finance Papers, (2009).

4. Z. Jiang, W. Zhou, D. Sornette, R. Woodard, K. Bastiaensen and P. Cauwels, Bubble Diagnosis and Prediction of the 2005-2007 and 2008-2009 Chinese stock market bubbles, Journal of Economic Behavior \& Organization, 74 (3) 149 (2010).

5. A. Krawiecki and J.A. Holyst, Stochastic resonance as a model for financial market crashes and bubbles, Physica A: Statistical Mechanics and its Applications, 317 (3-4) 597 (2003).

6. D. Sornette, R. Woodard and W.-X. Zhou, The 2006-2008 oil bubble: Evidence of speculation, and prediction, Physica A: Statistical Mechanics and its Applications, 10.1016/j.physa.2009.01.011, 388 (8) 1571 (2009).

7. T. Kaizoji and D. Sornette, Market bubbles and crashes, Encyclopedia of Quantitative Finance, (2010).

8. R. Woodard, D. Sornette and M. Fedorovsky, The Financial Bubble Experiment: Advanced Diagnostics and Forecasts of Bubble Terminations, Volume III, Quantitative Finance Papers, (2010).

9. T. Lux, Herd behaviour, bubbles and crashes, The Economic Journal, 105 (431) pp. 881 (1995).

10. F. Lillo, G. Bonanno and R.N. Mantegna, Variety of stock returns in normal and extreme market days: The August 1998 crisis, Empirical Science of Financial Fluctuations: pp. 77 (2002).

11. F. Lillo and R.N. Mantegna, Dynamics of a financial market index after a crash, Physica A: Statistical Mechanics and its Applications, 338 (1-2) 125 (2004).

12. V. Plerou, P. Gopikrishnan, B. Rosenow, L.A.N. Amaral, T. Guhr and H.E. Stanley, Random matrix approach to cross correlations in financial data, Physical Review E, 65 (6) pp. 66126 (2002).

13. B. Podobnik, D. Wang, D. Horvatic, I. Grosse and H. Stanley, Time-lag cross-correlations in collective phenomena, EPL (Europhysics Letters), 90 pp. 68001 (2010).

14. B. Podobnik, D. Horvatic, A.M. Petersen and H.E. Stanley, Crosscorrelations between volume change and price change, Proceedings of the National Academy of Sciences, 106 (52) pp. 22079 (2009)

15. B. Podobnik and H.E. Stanley, Detrended cross-correlation analysis: A new method for analyzing two nonstationary time series, Physical Review Letters, 100 (8) pp. 84102 (2008).

16. L. Xu, P.C. Ivanov, K. Hu, Z. Chen, A. Carbone and H. Stanley, Quantifying signals with power-law correlations: a comparative study of detrending and moving average techniques, Phys. Rev. E, 71 pp. 051101 (2005).
17. P.A. Reigneron, R. Allez and J.P. Bouchaud, Principal Regression Analysis and the index leverage effect, Arxiv preprint arXiv:1011.5810, (2010).

18. D. Harmon, M.A.M. de Aguiar, D.D. Chinellato, D. Braha, I.R. Epstein and Y. Bar-Yam, Predicting economic market crises using measures of collective panic, Arxiv preprint arXiv:1102.2620, (2011).

19. D.Y. Kenett, Y. Shapira, A. Madi, S. Bransburg-Zabary, G. GurGershgoren and E. Ben Jacob, Index Cohesive Force Analysis Reveals That the US Market Became Prone to Systemic Collapses Since 2002, PLoS ONE, 6 (4) pp. e19378, (2011).

20. D.Y. Kenett, Y. Shapira and E. Ben-Jacob, RMT assessments of market latent information embedded in the stocks' raw, normalized, and partial correlations, Hindawi Journal of Probability and Statistics, 2009: pp. 249370, 10.1155/2009/249370, (2009).

21. Y. Shapira, D.Y. Kenett and E. Ben-Jacob, The Index cohesive effect on stock market correlations, The European Physical Journal B, 72 (4): pp. 657 (2009).

22. D.Y. Kenett, M. Tumminello, A. Madi, G. Gur-Gershgoren, R.N. Mantegna and E. Ben-Jacob, Dominating Clasp of the Financial Sector Revealed by Partial Correlation Analysis of the Stock Market, PLoS ONE, 5 (12): pp. e15032 (2010).

23. R.N. Mantegna and H.E. Stanley, An Introduction to Econophysics: Correlation and Complexity in Finance, Cambridge, UK: Cambridge University Press, 2000.

24. K. Baba, R. Shibata and M. Sibuya, Partial correlation and conditional correlation as measures of conditional independence, Australian \& New Zealand Journal of Statistics, 46 (4) 657 (2004).

25. L. Laloux, P. Cizeau, J.P. Bouchaud and M. Potters, Noise dressing of financial correlation matrices, Physical Review Letters, 83 (7) 1467 (1999).

26. J.P. Bouchaud and M. Potters, Theory of Financial Risk and Derivative Pricing: Cambridge University Press, 2003.

27. J.P. Onnela, A. Chakraborti, K. Kaski and J. Kertesz, Dynamic asset trees and portfolio analysis, European Physical Journal B, 30 (3) 285 (2002).

28. J.P. Onnela, A. Chakraborti, K. Kaski and J. Kertesz, Dynamic asset trees and Black Monday, Physica A, 324 (1-2) 247 (2003).

29. M.J. Mauboussin, Revisiting market efficiency: the stock market as a Complex Adaptive System, Journal of Applied Corporate Finance 14 (4) 47 (2005).

30. Y. Demyanyk and O.V. Hemert, Understanding the Subprime Mortgage Crisis: pp. 1-33(2009).

31. P. Sieczk, D. Sornette and J.A. Holyst, The Lehman Brothers Effect and Bankruptcy Cascades, Swiss Finance Institute Research Paper 10-06 (2010).

32. J. Taylor, The financial crisis and the policy responses: An empirical analysis of what went wrong, NBER Working paper, (2009). 